\title{
SPLITTING OF ALGEBRAS BY FUNCTION FIELDS OF ONE VARIABLE
}

\author{
PETER ROQUETTE
}

To the memory of TADasi NaKayama

\section{§1. Introduction}

Let $K$ be a field and $\mathfrak{B}(K)$ the Brauer group of $K$. It consists of the similarity classes of finite central simple algebras over $K^{1{ }^{1}}$ ( For any field extension $F / K$ there is a natural mapping $\mathfrak{B}(K) \rightarrow \mathfrak{B}(F)$ which is obtained by assigning to each central simple algebra $A / K$ the tensor product $A \underset{K}{\otimes} F$ which is a central simple algebra over $F$. The kernel of this map is the relative Brauer group $\mathfrak{B}(F / K)$, consisting of those $A \in \mathfrak{B}(K)$ which are split by $F$.

If $F / K$ is finite algebraic, the investigation of $B(F / K)$ is part of the general theory of central simple algebras. In particular, if the ground field $K$ is a number field or a local number field, ${ }^{2)}$ the relative Brauer group $\$ B(F / K)$ can then explicitly be determined, using class field theory.

In this paper, we propose to investigate $\mathfrak{B}(F / K)$ in the case where $F / K$ is a function field of one variable. ${ }^{3)}$ Our results will give a complete description of $\mathfrak{B}(F / K)$ if $K$ is a local number field.

For any transcendental field extension $F / K$, let $p$ be a place of $F / K$ such that the image field $F \mathfrak{p}$ is algebraic over $K^{4}{ }^{4}$ Any central simple algebra $A / K$ which is split by $F$ is also split by $F$, as we have shown in an earlier

Received July 19, 1965.

1) For the general theory of central simple algebras see e.g. Deuring [6], chap. IV and V, or Artin-Nesbitt-Thrall [2], chap. V-VIII.

2) A field $K$ is called a number field if it is a finite-dimensional extension of the field $\mathbf{Q}$ of rational numbers. A field $K$ is called a local number field if it is the completion of a number field $k$ with respect to a non-trivial valuation of $K$. This is the case if and only if $K$ is either the field of real numbers or the field of complex numbers (archimedean case), or if $K$ is a finite-dimensional extension of the rational $p$-adic field $\mathbf{Q}_{p}$, for some prime number $p$.

3) A field extension $F / K$ is called a function field of one variable, if $F / K$ is finitely generated, $K$ is algebraically closed in $F$, and the degree of transcendency of $F / K$ is 1 .

4) For the general theory of places see e.g. Zariski-Samuel [15], vol. II, chap. VI. 
paper. ${ }^{5} \quad$ That is, we have $\mathfrak{B}(F / K) \subset \mathfrak{B}(F \mathfrak{p} / K)$. Let us put

$$
\widetilde{\mathfrak{B}}(F / K)=\bigcap_{\mathfrak{p}} \mathfrak{B}(F \mathfrak{p} / K),
$$

$\mathfrak{p}$ ranging over the places of $F / K$ such that $F \mathfrak{p} / K$ is algebraic. We then have

$$
\mathfrak{B}(F / K) \subset \widetilde{\mathfrak{B}}(F / K) .
$$

If $F / K$ is a separable function field of one variable, we shall show in $\S 3$ that the factor group $\frac{\widetilde{B}(F / K)}{B(F / K)}$ can be described by a certain cohomological invariant $X(F / K)$ which is connected with the one-dimensional Galois cohomology of the idele class group.

The interpretation of this result is as follows: As we have said above, the investigation of $B(L / K)$ for an algebraic field extension $L$ of $K$ is part of the classical theory of central simple algebras. Hence we may regard $\widetilde{B}(F / K)$, which concerns only Brauer groups of algebraic extensions $F \mathfrak{p} / K$, as essentially known, in particular if the ground field is a number field or a local number field. Hence the invariant $X(F / K)$, which is explicitely defined in $\S 3$, will describe the deviation of the group $\mathfrak{B}(F / K)$ from the (known) group $\widetilde{B}(F / K)$.

In the special case where $K$ is a local number field we shall see that $X(F /$ $K)=1$. In the archimedean case, this will follow from the results of Witt [12] while in the non-archimedean case we shall refer to the corresponding results of Tate [11]. This then shows that $\mathfrak{R}(F / K)=\widetilde{\mathfrak{B}}(F / K)$. On the other hand, the known structure of $\mathfrak{B}(K)$ for local number fields permits to determine $\widetilde{B}(F / K)$ explicitly. We then will obtain the following result which constitutes the main result of this paper:

THEOREM 1. Let $F / K$ be a function field of one variable over a local number field $K$. Let $d(F / K)$ be the smallest positive integer which is a degree of a divisor of $F / K$.

Then:

The group $\mathfrak{B}(F / K)$ is cyclic of order $d(F / K)$; it consists of all $A \in \mathfrak{B}(K)$ whose Schur index divides $d(F / K)$.

As to global number fields $K$ as ground fields, we shall show by examples

5) [9], page 428 , prop. 8 . 
that the equality $\mathfrak{B}(F / K)=\widetilde{\mathfrak{B}}(F / K)$ is not true in general. This case has still to be investigated.

\section{$\S 2$. The cohomological language}

Let $\bar{K} / K$ be a finite Galois extension of $K$, with Galois group $G=G(\bar{K} / K)$. As shown in the theory of crossed product algebras, we have

$$
\mathfrak{B}(\bar{K} / K)=H^{2}\left(G, \bar{K}^{\times}\right)^{6}
$$

where $\bar{K}^{\times}$denotes the multiplicative group of the field $K$. Here, $H^{2}\left(G, \bar{K}^{\times}\right)$ denotes the second cohomology group of $G$ in the multiplicative group of $\bar{K}$."

If $L / K$ is any field extension and $\bar{L}=L \bar{K}$ a field compositum of $L$ with $\bar{K}$, then the subgroup

$$
G_{L}=G(\bar{K} / L \cap \bar{K})
$$

of $G$ can be regarded as the Galois group of $\bar{L} / L$. The restriction from $G$ to $G_{L}$ together with the inclusion map $\bar{K}^{\times} \subset L^{\times}$gives a cohomology map

$$
H^{2}\left(G, \bar{K}^{\times}\right) \rightarrow H^{2}\left(G_{L}, \bar{L}^{\times}\right) .
$$

On the other hand, the map $\mathfrak{B}(K) \rightarrow \mathfrak{B}(L)$ described in $\S 1$ induces a map

$$
\mathfrak{B}(\bar{K} / K) \rightarrow \mathfrak{B}(\bar{L} / L) .
$$

In the theory of crossed products it is shown that the two maps (2) and (3) coincide after the identification (1) and the corresponding identification for $L / L .^{8)} \quad$ The kernel of (3) consists of those algebras over $K$, which are split by $\bar{K}$ and $L$. That is, this kernel is $\mathfrak{B}(\bar{K} / K) \cap \mathfrak{B}(L / K)$.

Hence :

$$
\mathfrak{B}(\bar{K} / K) \cap \mathfrak{B}(L / K)=\text { kernel } H^{2}\left(G, \bar{K}^{\times}\right) \rightarrow H^{2}\left(G_{L}, \bar{L}^{\times}\right) .
$$

If $L=F$ is a separable function field of one variable, then $F$ has a separable place $\mathfrak{p}$; hence we may choose $\bar{K}$ so as to contain $F$ p. As said in $\S 1$, $\mathfrak{B}(F / K)$ $\subset \mathfrak{B}(F \mathfrak{p} / K) \subset \mathfrak{B}(\bar{K} / K)$. On the other hand, $F$ is linearly disjoint to $\bar{K}$ over $K$ and hence $G_{F}=G$ can be regarded as the Galois group of $\bar{F}=F \cdot \bar{K}$ over $F$.

6) See e.g. the books mentioned in ${ }^{1)}$. For another approach see Serre [10], chap. $X, \S 5-6$.

7) For the general cohomology theory we refer to [10] chap. VII, or Cartan-Eilenberg [3], or Artin [1].

8) Deuring [6], page 61, Satz 1. 
Hence :

Proposition 1. Let $F / K$ be a separable function field of one variable. Then there exists a finite Galois extension $\bar{K} / K$ such that $\mathfrak{B}(F / K) \subset \mathfrak{B}(\bar{K} / K)$. If this is so, then

$$
\mathfrak{B}(F / K)=\operatorname{kernel} H^{2}\left(G, \bar{K}^{\times}\right) \rightarrow H^{2}\left(G, \bar{F}^{\times}\right) .
$$

Next we shall give a cohomological interpretation of $\widetilde{\mathfrak{B}}(F / K)$.

Let $\mathfrak{p}$ be a place of $F / K$ and $F \mathfrak{p}$ its image field. Let $F \mathfrak{p} \cdot \bar{K}$ be a field compositum of $F p$ and $\bar{K}$ over $K$ and denote by $G_{\mathfrak{p}}$ the group of $\bar{K}$ over $F \mathfrak{p} \cap \bar{K}$. From (4) we obtain:

$$
\mathfrak{B}(\bar{K} / K) \cap \mathfrak{B}(F \mathfrak{p} / \bar{K})=\text { kernel } H^{2}\left(G, \bar{K}^{\times}\right) \rightarrow H^{2}\left(G_{\mathfrak{p}},(F \mathfrak{p} \cdot \bar{K})^{\times}\right) .
$$

Let now $p$ range over all places of $F / K$ and

$$
H^{2}\left(G, \bar{K}^{\times}\right) \rightarrow \prod_{\mathfrak{p}} H^{2}\left(G_{\mathfrak{p}},(F \mathfrak{p} \cdot \bar{K})^{\times}\right)
$$

be the map which in each component of the direct product induces the map mentioned in (5). Its kernel is the intersection of the kernels in (5). Hence

$$
\mathfrak{B}(\bar{K} / K) \cap \widetilde{\mathfrak{B}}(F / K)=\operatorname{kernel} H^{2}\left(G, \bar{K}^{\times}\right) \rightarrow \prod_{\mathfrak{p}} H^{2}\left(G_{\mathfrak{p}},(F \mathfrak{p} \cdot \bar{K})^{\times}\right)
$$

If we choose $\bar{K}$ such that it contains $F p$ for some $p$, which is possible if $F / K$ is separable, then $\widetilde{\mathfrak{B}}(F / K)$ is contained in $\mathfrak{B}(\bar{K} / K)$ and hence we may replace the intersection on the left hand side of $(6)$ by $\widetilde{B}(F / K)$.

On the right hand side of (6), the image group is a direct product of cohomology groups with respect to various subgroups $G_{\mathrm{p}}$ of $G$. However, this group can be interpreted as a cohomology group of $G$ in a certain group $\bar{W}=\bar{W}(\bar{F} / \bar{K})$, as follows.

For a given prime $\mathfrak{p}$, the field compositum $F \mathfrak{p} \cdot \bar{K}$ is in general not uniquely determined. There may be several inequivalent field composita of $F$ p with $\bar{K}$ over $K$. Let $\bar{p}$ range over the primes of $\bar{F} / \bar{K}$ which lie above $\mathfrak{p}$ (we then write $\bar{p} \mid \mathfrak{p}$.). It is well known that the inequivalent field composita of $F$ w with $\bar{K}$ correspond $1-1$ to the $\overline{\mathfrak{p}} \mid \mathfrak{p}$. For any $\overline{\mathfrak{p}} \mid \mathfrak{p}$, the image field $\bar{F} \overline{\mathfrak{p}}$ contains $F \overline{\mathfrak{p}}$, which is $K$-isomorphic to $F$ p under the map

$$
a \mathfrak{p} \rightarrow a \overline{\mathfrak{p}} \quad(a \in F) .
$$

We have 


$$
\bar{F} \overline{\mathfrak{p}}=F \overline{\mathrm{p}} \cdot \bar{K},
$$

and this is the field compositum belonging to $\overline{\mathfrak{D}} .^{9)}$

We now form the direct product $\prod_{\overline{\mathfrak{p}} \mid \mathrm{p}} \bar{F} \bar{p}$. Since the $\bar{F} \bar{p}$ are all the inequivalent field composita of $F p$ and $\bar{K}$, we have a natural isomorphism

$$
F \mathfrak{K} \underset{K}{\otimes} \bar{K}=\prod_{\overline{\mathfrak{p}} \mid \mathfrak{p}} \bar{F} \overline{\mathfrak{p}}
$$

which is obtained by mapping $\bar{K}$ diagonally into $\prod_{\bar{p}} \bar{F} \overline{\mathfrak{p}}(\bar{K}$ is contained in each $\bar{F} \overline{\mathfrak{p}})$ and by mapping

$$
a \mathfrak{p} \rightarrow \prod_{\overline{\mathfrak{p}} \mid \mathfrak{p}} a \overline{\mathfrak{p}} \quad(a \in F) .
$$

The Galois group $G$ acts naturally on $F p \otimes \bar{K}$ (on the right factor) and hence on the direct product on the right hand side of (7), thereby permuting the factors $\bar{F} \bar{p}$ transitively. If $\bar{p} \mid p$ is fixed and $G_{\bar{p}}$ denotes the subgroup of $G$ leaving the elements of $\bar{F} \bar{p}$ fixed, then we may write

$$
F \mathfrak{p} \underset{K}{\otimes} \bar{K}=\prod_{\sigma \in G \bmod G_{\bar{p}}}(\bar{F} \bar{p})^{\sigma} .
$$

Let $\bar{W}_{\mathfrak{p}}$ be the group of units of the algebra $F \mathfrak{p} \otimes \bar{K}$. We obtain

$$
\bar{W}_{\mathfrak{p}}=\prod_{\sigma \bmod G_{\bar{p}}}(\bar{F} \bar{p})^{\times \sigma} .
$$

Shapiros lemma from cohomology theory ${ }^{101}$ now shows that

$$
H^{i}\left(G, \bar{W}_{\mathfrak{p}}\right)=H^{i}\left(G_{\bar{p}},(\bar{F} \bar{p})^{\times}\right) \quad(i \geq 0) .
$$

This isomorphism is obtained by the restriction of $G$ to the subgroup $G_{\bar{p}}$, followed by the projection $\bar{W}_{\mathfrak{p}} \rightarrow(\bar{F} \bar{p})^{\times}$.

Observe that on the right hand side in (10) we have one fixed compositum $\bar{F} \overline{\mathfrak{p}}=F \overline{\mathfrak{p}} \cdot \bar{K}$ of $F \mathfrak{p}$ and $\bar{K}$. This may take the place of what we have denoted by $F \mathfrak{p} \cdot \bar{K}$ in (5). The diagonal imbedding $\bar{K}^{\times} \rightarrow \bar{W}_{\mathfrak{p}}$ followed by the projection $\bar{W}_{\mathfrak{p}} \rightarrow(\bar{F} \bar{p})^{\times}$is precisely the natural injection $\bar{K}^{\times} \rightarrow(\bar{F} \bar{p})^{\times}=(F \bar{p} \cdot \bar{K})^{\times}$. Hence we obtain from (5) and (10) (for $i=2$ ) that

$$
\widetilde{\mathfrak{B}}(\bar{K} / K) \cap \mathfrak{B}(F \mathfrak{p} / K)=\text { kernel } H^{2}\left(G, \bar{K}^{\times}\right) \rightarrow H^{2}\left(G, \bar{W}_{\mathfrak{p}}\right) .
$$

9) Chevalley [5], page 92, theorem 3.

10) [10], page 125, exercice, 
Now let us put all places $\mathfrak{p}$ of $F / K$ together:

$$
\bar{W}=\prod_{\mathfrak{p}} \bar{W}_{\mathfrak{p}}=\prod_{\overline{\mathfrak{p}}}(\bar{F} \overline{\mathfrak{p}})^{\times}
$$

$G$ acts on $\bar{W}$ componentwise on each $\bar{W}_{\mathfrak{p}}$. We have

$$
H^{i}(G, \bar{W})=\prod_{\mathfrak{p}} H^{i}\left(G, \bar{W}_{\mathfrak{p}}\right) \quad(i \geqq 0)
$$

and we obtain:

Proposition 2. Let $F / K$ be a separable function field of one variable. Then there is a finite Galois extension $\bar{K} / K$ such that $\mathfrak{B}(F / K) \subset \mathfrak{B}(\bar{K} / K)$. If this is so, we have

$$
\widetilde{\mathfrak{B}}(F / K)=\operatorname{kernel} H^{2}\left(G, \bar{K}^{\times}\right) \rightarrow H^{2}(G, \bar{W}),
$$

where

$$
\bar{W}=\prod_{\overline{\mathfrak{p}}}(\bar{F} \overline{\mathrm{p}})^{\times}
$$

( $\bar{p}$ ranging over the places of $\bar{F} / \bar{K}$ ), and $G$ acts on $\bar{W}$ naturally as described above.

\section{§ 3. The kernel theorem}

Let $F / K$ be a function field of one variable and $\bar{K} / K$ a finite Galois extension with group $G$. According to propositions 1 and 2, we shall study in this $\S 3$ the maps

$$
H^{2}\left(G, \bar{K}^{\times}\right) \rightarrow H^{2}\left(G, \bar{F}^{\times}\right)
$$

and

$$
H^{2}\left(G, \bar{K}^{\times}\right) \rightarrow H^{2}(G, \bar{W})
$$

described in $\S 2$ and we shall compare their kernels.

We introduce the following notations:

$D$ the divisor group of $F / K$

$H$ the group of principal divisors in $D$

$C D=D / H$ the divisor class group

$J$ the idèle group of $F / K$

$C J=J / F^{\times}$the group of idele classes

$U$ the group of idèle units in $J$

$C U=U F^{\times} / F^{\times}=U / K^{\times}$the idèle unit classes.

As to the definitions, $D$ is defined to be the free abelian multiplicative 
group generated by the prime divisors (places) $p$ of $F / K$. Hence every divisor $a \in D$ is a product

$$
\mathfrak{a}=\prod_{\mathfrak{p}} \mathfrak{p}^{a(\mathfrak{p})}
$$

with uniquely determined integers $a(p)$ such that $a(p)=0$ for all but a finite number of $\mathfrak{p}$.

Let $w_{\mathfrak{p}}$ be the additive normalized valuation of $F$ belonging to $\mathfrak{p}$. The principal divisor for $a \in F^{\times}$is

$$
(a)=\prod_{\mathfrak{p}} \mathfrak{p}^{w \mathfrak{p}(a)}
$$

$H$ is defined to be the image of the map $a \rightarrow(a)$ from $F^{\times}$into $D$. The kernel of this map is $K^{\times}$, so that the sequence

$$
1 \rightarrow K^{\times} \rightarrow F^{\times} \rightarrow H \rightarrow 1
$$

is exact.

$J$ is defined to consist of all functions $p \rightarrow \alpha(p)$, defined on the primes $p$ of $F / K$, with values $\alpha(\mathfrak{p})$ in the multiplicative group of $F_{\mathfrak{p}}$, the $\mathfrak{p}$-adic completion of $F$ with respect to $p$.

These functions $\alpha$ have to satisfy the finiteness condition that $w_{p}(\alpha(p)) \neq 0$ for all but a finite number of $\mathfrak{p}^{11}{ }^{11} \quad$ There is a mapping $J \rightarrow D$ obtained by assigning to each $\alpha \in J$ its divisor $(\alpha)=\prod_{\mathfrak{p}} p^{w_{p}(\alpha(p) \prime}$.

This mapping is epimorphic; its kernel is called $U$, so that the sequence

$$
1 \rightarrow U \rightarrow J \rightarrow D \rightarrow 1
$$

is exact.

There is a mapping $F^{\times} \rightarrow J$ obtained by assigning to each $a \in F^{\times}$the idèle $\alpha_{a}$ given by $\alpha_{a}(\mathfrak{p})=a$, for all $\mathfrak{p}$ (diagonal imbedding). This mapping is monomorphic and we identify $F^{\times}$with its image in $J$. This identification is coherent with the mappings $F^{\times} \rightarrow D$ and $J \rightarrow D$, i.e. we have $(a)=\left(\alpha_{a}\right)$. In other words, the diagram

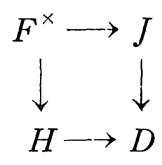

is commutative.

11) By continuity, the valuation $w_{p}$ of $F$ extends uniquely to a valuation of the completion $F_{\mathfrak{p}}$, and this extension is again denoted by $w_{\mathfrak{p}}$. 
From the above definitions and discussions it follows that the diagram

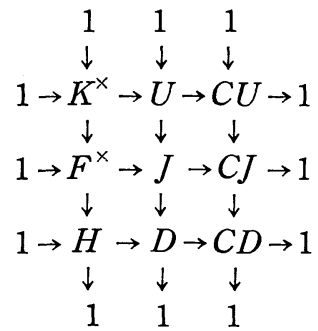

in which the arrows denote the natural maps in question, is commutative with exact rows and columns.

For the function field $\bar{F} / \bar{K}$ we have a similar diagram whose corresponding groups will be denoted by $\bar{D}, \bar{H}, C D, \bar{J}$, etc:

$$
\begin{aligned}
& \begin{array}{lll}
1 & 1 & 1
\end{array} \\
& 1 \rightarrow \frac{\downarrow}{\bar{K}^{\times}} \rightarrow \frac{\downarrow}{\bar{U}} \rightarrow \frac{\downarrow}{\bar{C} U} \rightarrow 1
\end{aligned}
$$

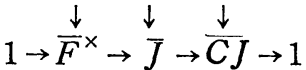

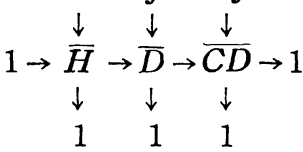

As said in $\S 2$, the Galois group $G=G(\bar{K} / K)$ can be regarded as the Galois group of $\bar{F} / F$, since $F$ and $\bar{K}$ are linearly disjoint over $K$. Hence $G$ acts on $\bar{F}^{\times}$. Also, $G$ acts on all the other groups of our diagram, as follows.

$G$ acts on the primes $\bar{p}$ of $\bar{F}$ : If $w_{\bar{p}}$ is the additive normalized valuation of $\bar{F}$ belonging to $\overline{\mathfrak{p}}$ then $\overline{\mathfrak{p}}^{\sigma}$ is defined by

$$
w_{\overline{\mathfrak{p}} \sigma}\left(a^{\sigma}\right)=w_{\overline{\mathfrak{p}}}(a) \quad(a \in F, \sigma \in G) .
$$

The map $\sigma: \bar{F} \rightarrow \bar{F}$ is continuous if $\bar{F}$ as the domain of this map is topologized by $w_{\overline{\mathfrak{p}}}$, and it is topologized by $w_{\bar{p} \sigma}$ if considered as the range of $\sigma$. Hence $\sigma$ extends, by continuity, uniquely to a map $\sigma: \bar{F}_{\bar{p}} \rightarrow \bar{F}_{\bar{p} \sigma}$ of the corresponding completions. According to these maps, $G$ acts on $\bar{J}$, namely:

$$
\alpha^{\sigma}\left(\overline{\mathfrak{p}}^{\Im}\right)=\alpha(\overline{\mathfrak{p}})^{\sigma} \quad(\alpha \in \bar{J}, \sigma \in G) .
$$

By definition, it is clear that the maps

$$
\bar{F}^{\times} \rightarrow \bar{J}
$$


(diagonal imbedding) and

$$
\bar{J} \rightarrow \bar{D}
$$

(divisor map) are $G$-permissible. Hence all the other maps of our diagram, being based on the two maps mentioned above, are $G$-permissible, $G$ acting on the groups of the diagram in the natural way. In other words: our diagram is $G$-permissible.

In particular, for each group $\bar{M}$ of our diagram we can form the cohomology groups $H^{i}(G, \bar{M})$, and for each exact row or column $1 \rightarrow \bar{M}_{1} \rightarrow \bar{M}_{2} \rightarrow \bar{M}_{3} \rightarrow 1$ of our diagram we obtain a cohomological connecting map $H^{i}\left(G, \bar{M}_{3}\right) \rightarrow H^{i+1}(G$, $\bar{M}_{1}$ ).

From the lower horizontal sequence of the diagram we thus obtain a cohomology map

$$
H^{i}(\overline{C D}) \rightarrow H^{i+1}(\bar{H})
$$

From the left vertical sequence we obtain also

$$
H^{i+1}(\bar{H}) \rightarrow H^{i+2}\left(\bar{K}^{\times}\right)
$$

which combined with the map above yields a map

$$
h^{i}: H^{i}(\overline{C D}) \rightarrow H^{i+2}\left(\bar{K}^{\times}\right) .
$$

Similarly, using first the right vertical sequence and then the upper horizontal sequence of the diagram we obtain another map

$$
g^{i}: H^{i}(\overline{C D}) \rightarrow H^{i+2}\left(\bar{K}^{\times}\right) .
$$

It is known from general cohomology theory ${ }^{12)}$ that both maps $h^{i}$ and $g^{i}$ differ only by a sign; in particular, both maps have the same kernel and the same image.

Let us investigate these maps in the case $i=0$.

Investigation of $h^{0}$ :

By definition, $h^{0}$ is obtained by considering the left lower corner of the diagram, namely :

12) Cartan-Eilenberg [3], page 56, prop. 2.1 . 


$$
\begin{aligned}
& 1 \\
& \frac{\downarrow}{K^{\times}} \\
& \frac{\downarrow}{F^{\times}} \\
& 1 \rightarrow \stackrel{\downarrow}{\bar{H}} \rightarrow \bar{D} \rightarrow \overline{C D} \rightarrow 1 \\
& \downarrow
\end{aligned}
$$

This portion of our diagram gives the two maps

$$
H^{0}(\overline{C D}) \rightarrow H^{1}(\bar{H})
$$

and

$$
H^{1}(\bar{H}) \rightarrow H^{2}\left(\bar{K}^{\times}\right)
$$

the composite of which is $h^{0}$.

We begin by observing that

$$
H^{1}\left(\bar{F}^{\times}\right)=1
$$

and

$$
H^{1}(\bar{D})=1 \text {. }
$$

The first of these formulae is well known as the celebrated 'Hilbert theorem 90'. The second follows from the fact that $\bar{D}$ is the free abelian group generated by the primes $\bar{p}$ of $\bar{F} / \bar{K}$ which are only permuted under $G .{ }^{13)}$

From (13) it follows, using the exactness of the column of our diagram portion, that

$$
H^{1}(\bar{H}) \rightarrow H^{2}\left(\bar{K}^{\times}\right) \text {is monomorphic. }
$$

Similarly, from (14) it follows that

$$
H^{0}(\overline{C D}) \rightarrow H^{1}(\bar{H}) \text { is epimorphic. }
$$

Putting both statements together we obtain

$$
\text { image } \begin{aligned}
\left(h_{0}\right) & =\text { image } H^{1}(\bar{H}) \rightarrow H^{2}\left(\bar{K}^{\times}\right) \\
& =H^{1}(\bar{H}) .
\end{aligned}
$$

On the other hand,

$$
\text { image } H^{1}(\bar{H}) \rightarrow H^{2}\left(\bar{K}^{\times}\right)=\text {kernel } H^{2}\left(\bar{K}^{\times}\right) \rightarrow H^{2}\left(\bar{F}^{\times}\right)
$$

13) Seẹ e.g. [9], page 437, 
so that we finally obtain

$$
\text { image }\left(h^{0}\right)=\text { kernel } H^{2}\left(\bar{K}^{\times}\right) \rightarrow H^{2}\left(\bar{F}^{\times}\right) \text {. }
$$

Investigation of $g^{0}$ :

Now we have to consider the right upper corner of our diagram:

$$
1 \rightarrow \bar{K}^{\times} \rightarrow \bar{U} \rightarrow \begin{gathered}
1 \\
\downarrow \\
\overline{C U} \rightarrow 1 \\
\downarrow \\
\overline{C J} \\
\frac{\downarrow}{C D} \\
\downarrow \\
1
\end{gathered}
$$

$g^{0}$ is the composite of the two maps

$$
H^{0}(\overline{C D}) \rightarrow H^{1}(\overline{C U})
$$

and

$$
H^{1}(\overline{C U}) \rightarrow H^{2}\left(\bar{K}^{\times}\right) .
$$

First we have, in analogy to (13), the formula

$$
H^{1}(\bar{U})=1 .
$$

Proof. Let $\bar{U}_{\bar{p}}$ be the group of $\bar{p}$-adic units in the $\bar{p}$-adic completion $\bar{F}_{\bar{p}}$ of $\bar{F}$. By definition, $\bar{U}$ is the direct product

$$
\bar{U}=\prod_{\bar{p}} \bar{U}_{\bar{p}}
$$

Each place $\bar{p}$ induces an epimorphic map

$$
\bar{U}_{\overline{\mathfrak{p}}} \rightarrow(\bar{F} \bar{p})^{\times} .
$$

These maps define an epimorphic map

$$
\bar{U} \rightarrow \bar{W}
$$

where $\bar{W}$ is the direct product of the $(\bar{F} \bar{p})^{\times}$as in $\S 2$. By comparing the definitions of the actions of $G$ on $\bar{U}$ (as part of $J$ ) and on $\bar{W}$ (see $\S 2$ ) we see that this map is $G$-permissible.

Let $\bar{V}$ be the kernel, so that

$$
1 \rightarrow \bar{V} \rightarrow \bar{U} \rightarrow \bar{W} \rightarrow 1
$$


is exact. We shall show in a moment that

$$
H^{i}(G, \bar{V})=1 \quad(i \geqq 1) .
$$

This shows that $\bar{U} \rightarrow \bar{W}$ induces an isomorphism

$$
H^{i}(G, \bar{U})=H^{i}(G, \bar{W}) \quad(i \geqq 1) .
$$

Using (12) and (10) we obtain

$$
H^{i}(G, \bar{U})=\prod_{\mathfrak{p}} H^{i}\left(G_{\bar{p}},(\bar{F} \bar{p})^{\times}\right) \quad(i \geqq 1)
$$

where $\mathfrak{p}$ ranges over the places of $F / K$ and $\bar{p}$ denotes always a fixed extension of $\mathfrak{v}$ to $\bar{F} / \bar{K}$. For $i=1$, the right hand side of $(19)$ is 1 , using Hilberts theorem 90 for each field $\bar{F} \bar{p}$. Hence (17).

Proof of (18). Let $\bar{V}_{\bar{p}}$ be the kernel of the map $\bar{U}_{\bar{p}} \rightarrow(\bar{F} \bar{p})^{\times}$, consisting of the elements $a \in \bar{F}_{\overline{\mathfrak{p}}}$ with $a \overline{\mathfrak{p}}=1 . \quad$ Then $\bar{V}=\prod_{\overline{\mathfrak{p}}} V_{\overline{\mathfrak{p}}}$. Put $\bar{V}_{\mathfrak{p}}=\prod_{\overline{\mathfrak{p}} \mid \mathfrak{p}} \bar{V}_{\overline{\mathfrak{p}}}$. Then $\bar{V}=\prod_{\mathfrak{p}} \bar{V}_{\mathfrak{p}}$ is a $G$-permissible direct product. Hence

$$
H^{i}(G, \bar{V})=\prod_{\mathfrak{p}} H^{i}\left(G, \bar{V}_{\mathfrak{p}}\right) .
$$

From Shapiros lemma ${ }^{101}$ we infer that

$$
H^{i}\left(G, \bar{V}_{\mathfrak{p}}\right)=H^{i}\left(G_{\bar{p}}, \bar{V}_{\bar{p}}\right),
$$

$\overline{\mathfrak{p}}$ being a fixed extension of $\mathfrak{p}$. Hence we have to show that $H^{i}\left(G_{\bar{p}}, \bar{V}_{\bar{p}}\right)=1$ for $i \geqq 1$. Changing notation, this amounts to show the following

Lemмa. Let $F$ be a complete field with respect to a non-archimedean, discrete valuation $w_{\mathfrak{p}}$ with corresponding prime $\mathfrak{p}$. Let $V$ be the multiplicative subgroup of elements $a \in F$ with $a \mathfrak{p}=1$ (i.e. $w_{\mathfrak{p}}(a-1)>0$ ). If $G$ is a finite group of continuous automorphisms of $F$ whose induced action on the image field $F p$ is faithful, then

$$
H^{i}(G, V)=1 \quad(i \geqq 1) .
$$

This lemma is well known from local class field theory. For the proof see e.g. Witt [14], page 154, no. 2 or Serre [10], page 193, lemma 2.

Let us return to our original notation. We now have proved (17) which is, for the map $g^{0}$, the analogue to (13). The analogue to (14) would be $H^{1}(G$, $\overline{C J})=1$. This is not true in general (although we shall see later that it is true in the case where $K$ is a local number field). We therefore introduce the group 


$$
X=X(\bar{F} / \bar{K})=\text { kernel } H^{1}(G, \overline{C J}) \rightarrow H^{1}(G, \overline{C D}) .
$$

From the exactness of the column of our diagram portion we infer that

$$
\begin{aligned}
X & =\text { image } H^{1}(G, \overline{C U}) \rightarrow H^{1}(G, \overline{C J}) \\
& =H^{1}(G, \overline{C U}) / Y
\end{aligned}
$$

where

$$
Y=\text { image } H^{0}(G, \overline{C D}) \rightarrow H^{1}(G, \overline{C U}) .
$$

From (17) it follows that

$$
H^{1}(G, \overline{C U}) \rightarrow H^{2}\left(G, \bar{K}^{\times}\right) \text {is monomorphic. }
$$

Its image is the kernel of $H^{2}\left(G, \bar{K}^{\times}\right) \rightarrow H^{2}(G, \bar{U})$. Hence

$$
H^{1}(G, \bar{C} \bar{U}) \approx \text { kernel } H^{2}\left(G, \bar{K}^{\times}\right) \rightarrow H^{2}(G, \bar{U}) .
$$

In this isomorphism, the image $Y$ of $H^{0}(G, \overline{C D}) \rightarrow H^{1}(G, \overline{C U})$ corresponds to the image of $g^{0}$ (by definition of $g^{0}$ ). Hence we obtain from (21):

(22) The image of $g^{0}$ is contained in the kernel of $H^{2}\left(G, \bar{K}^{\times}\right) \rightarrow H^{2}(G, \bar{U})$ and the corresponding factor group is isomorphic to $X$.

Finally, we claim:

$$
\text { kernel } H^{2}\left(G, \bar{K}^{\times}\right) \rightarrow H^{2}(G, \bar{U})=\text { kernel } H^{2}\left(G, \bar{K}^{\times}\right) \rightarrow H^{2}(G, \bar{W}) .
$$

Proof. As shown in (19), the map $\bar{U} \rightarrow \bar{W}$ induces an isomorphism of cohomology groups. Hence the map $\bar{K}^{\times} \rightarrow \bar{U} \rightarrow \bar{W}$ induces a map $H^{2}\left(G, \bar{K}^{\times}\right) \rightarrow$ $H^{2}(G, \bar{W})$ which has the same kernel as $H^{2}\left(G, \bar{K}^{\times}\right) \rightarrow H^{2}(G, \bar{U})$. Q.e.d.

Observe that the map $\bar{K}^{\times} \rightarrow \bar{U} \rightarrow \bar{W}$ is the diagonal imbedding of $\bar{K}^{\times}$in $\bar{W}$ which we have considered in $\S 2$.

Now remember that the maps $h^{0}$ and $g^{0}$ have the same image, as mentioned above. Comparing (16), (22) and (23) we obtain therefore the following 'kernel theorem' :

TheOREM 2. Let $F / K$ be a function field of one variable and $\bar{K} / K$ a finite Galois extension with Galois group $G$. Then the kernel of $H^{2}\left(G, \bar{K}^{\times}\right) \rightarrow H^{2}\left(G, \bar{F}^{\times}\right)$ is contained in the kernel of $H^{2}\left(G, \bar{K}^{\times}\right) \rightarrow H^{2}(G, \bar{W})$ and the corresponding factor group is isomorphic to $X$, where $X$ is defined to be the kernel of $H^{1}(G, \overline{C J}) \rightarrow H^{1}(G$, $\overline{C D}$ ).

In particular, if $H^{1}(G, \overline{C J})=1$ then $X=1$ and therefore kernel $H^{2}\left(G, \bar{K}^{\times}\right) \rightarrow$ $H^{2}\left(G, \bar{F}^{\times}\right)=$kernel $H^{2}\left(G, \bar{K}^{\times}\right) \rightarrow H^{2}(G, \bar{W})$. 
Using propositions 1 and 2 of $\S 2$ we obtain as an immediate consequence:

Theorem 3. Let $F / K$ be a separable function field of one variable. Then the factor group $\widetilde{\mathfrak{B}}(F / K)$ modulo $\mathfrak{B}(F / K)$ can be cohomologically described as the group $X$ of theorem 2 , where $\bar{K} / K$ has to be chosen such that $\widetilde{B}(F / K) \subset \mathfrak{B}(\bar{K} / K)$.

(As mentioned in $\S 2$, the latter inclusion is true if $\bar{K}$ contains the image field $F \mathfrak{p}$ of a separable place $\mathfrak{p}$ of $F / K$.)

In particular, if the Galois cohomology of the idèle classes $\overline{C J}$ vanishes in dimension 1 , then $\widetilde{\mathfrak{B}}(F / K)=\mathfrak{B}(F / K)$.

\section{$\S 4$. Proof of theorem 1}

Now let $F / K$ be a function field of one variable over a local number field $K$. If the valuation of $K$ is non-archimedean, then there is a theorem of Tate which says that the Galois cohomology of the idèle classes vanishes in dimension ${ }^{14}{ }^{14)}$ Hence $\widetilde{B}(F / K)=\mathfrak{B}(F / K)$.

Now let the valuation of $K$ be archimedean. Then $K$ is either the field of complex numbers, or the field of real numbers. In the first case $K$ is algebraically closed and hence $\widetilde{\mathfrak{B}}(F / K)=\mathfrak{B}(F / K)=1$. In the second case, assume first that $F / K$ has a real place p. Then $F \mathfrak{p}=K, \widehat{\mathcal{B}}(F / K) \subset \mathfrak{B}(K / K)=1$, hence $\mathfrak{B}(F / K)=\mathfrak{B}(F / K)=1$. Secondly, if all places $\mathfrak{p}$ of $F / K$ are complex, then $F \mathfrak{p}$ $=\bar{K}$ is the field of complex numbers for all $\mathfrak{p}$. Hence $\mathbb{B}(F / K)=\mathfrak{B}(\bar{K} / K)=$ $\mathfrak{B}(K)$ is of order two, the only non-trivial element of $\mathfrak{B}(K)$ corresponding to the quaternion algebra over $K$. On the other hand, Witt has shown that if $F / K$ has no real places, then -1 is a sum of two squares in $F$, hence -1 is a norm of $F \bar{K} / F$, i.e. the quaternion algebra splits over $F^{15}$ ) This implies that $\mathfrak{B}(F / K)$ contains the quaternion algebra and is therefore equal to $\mathfrak{B}(K)$.

Hence, in any case, $\widetilde{B}\left(F_{\prime}^{\prime} K\right)=\mathfrak{B}(F / K) .^{16)}$

14) Tate [11], page $156-02$, line $2-5$.

15) Witt [12], page 7 Satz 2.

16) Using Witts results, it can be shown that Tates relation $H^{1}(G, \bar{C} \bar{J})=1$ holds also if $K$ is real and $\bar{K}$ complex. For, if one interprets Witts statement I' ([12], page 5) cohomologically, it says that the map $H^{2}\left(G, \bar{F}^{\times}\right) \rightarrow H^{2}(G, \bar{J})$ is injective. On the other hand, from our diagram in $\S 3$ we obtain an exact sequence $H^{1}(G, \bar{J}) \rightarrow H^{1}(G, \overline{C J}) \rightarrow H^{2}(G$, $\bar{F} \times) \rightarrow H^{2}(G, \bar{J})$ and we have $H^{1}(G, \bar{J})=1$ from Hilberts theorem 90 for the completions $\overline{F_{\bar{p}}}$. Hence $H^{1}(G, \bar{C} J)=1$. 
In order to complete the proof of theorem 1 we have to describe the group $\widetilde{\mathfrak{S}}(F / K)$.

Consider first the non-archimedean case. As is well known from local class field theory, the Brauer group $\mathfrak{B}(K)$ is isomorphic to the additive group $\mathbf{Q} / \mathbf{Z}$ of rational numbers modulo integers. ${ }^{17)}$ The isomorphism

$$
\mathfrak{B}(K) \approx \mathbf{Q} / \mathbf{Z}
$$

is obtained by assigning to each central simple algebra $A / K$ its Hasse invariant $\operatorname{inv}_{K}(A)$. If $L / K$ is a finite algebraic extension field, then $\operatorname{inv}_{\iota}(A \underset{K}{\otimes} L)=$ $(L: K) \cdot \operatorname{inv}_{K}(A)$. In particular, $L$ splits $A$ if and only if $(L: K)$ is a multiple of the order of $A$ in $\mathfrak{B}(K)$. In other words $\mathfrak{B}(L / K)$ consists of all those $A \in \mathfrak{B}(K)$ for which $A^{(L: K)}=1$. The group structure of $\mathbf{Q} / \mathbf{Z}$ implies moreover that $\mathfrak{B}(L / K)$ is cyclic of order $(L: K)$.

In particular, $\mathfrak{B}(F \mathfrak{p} / K)$ is cyclic of order $(F \mathfrak{p}: K)=\operatorname{deg}(\mathfrak{p})$, and $\mathfrak{B}(F \mathfrak{p} / K)$ consists of all elements $A \in \mathfrak{B}(K)$ with $A^{\operatorname{deg}(\mathfrak{p})}=1$. Taking the intersection for all $\mathfrak{p}$, we see that if

$$
0<d(F / K)=\underset{\mathfrak{p}}{\operatorname{gcd}} \operatorname{deg}(\mathfrak{p})
$$

then $\widetilde{B}(F / K)$ is cyclic of order $d(F / K)$ and consists of all elements $A \in \mathfrak{B}(K)$ with $A^{d(F / K)}=1$.

If $\mathfrak{a}=\prod_{\mathfrak{p}} \mathfrak{p}^{a(\mathfrak{p})}$ is a divisor of $F / K$ then $\operatorname{deg}(\mathfrak{a})=\sum_{\mathfrak{p}} a(\mathfrak{p}) \cdot \operatorname{deg}(\mathfrak{p})$ is a linear combination of the degrees deg $(p)$, hence a multiple of $d(F / K)$, and conversely. Hence $d(F / K)$ can be characterized as the least positive degrees of divisors of $F / K$.

This proves theorem 3 in the non-archimedean case, if one uses the fact (proved by studying the Hasse invariant as above) that the Schur index of any $A \in \mathfrak{B}(K)$ is equal to its order in $\mathfrak{B}(K)$.

In the archimedean real case, we have

$$
\mathfrak{B}(K) \approx \frac{1}{2} \mathbf{Z} / \mathbf{Z}
$$

If one defines the Hasse invariant of the quaternion algebra to be $\frac{1}{2}$ modulo $\mathbf{Z}$, then the above considerations carry over verbally in order to prove theorem 1.

17) See e.g. Deuring [6], page 112, Satz 3 . 
In the archimedean complex case, there is nothing to prove.

\section{$\S 5$. Some additional remarks}

(a) Examples of function fields $F / K$ over a number field $K$ for which $B(F / K)$ $\neq \widetilde{\mathfrak{B}}(F / K)$ :

Let $K$ be a number field and $F / K$ be a function field of one variable which is of genus 0 but not rational. It has been shown by Witt [13] that $F=F(A)$ is a generic splitting field of a certain quaternion algebra $A$ over $K$ which is uniquely determined by $F$. Then $\mathfrak{B}(F / K)$ is of order 2 , the only non-trivial element of $\mathfrak{B}(F / K)$ being $A$; this follows also from our general theory of generic splitting fields ${ }^{18)}$. Let $q$ range over the primes of $K$ including the primes at infinity. Let $M$ be the set of primes $q$ at which $A$ is ramified, i.e. for which the $q$-adic Hasse invariant $\operatorname{inv}_{\mathfrak{q}}(A) \equiv \frac{1}{2} \bmod \mathbf{z}$. According to the Hasse sum formula $\sum_{\mathfrak{q}} \operatorname{inv}_{\mathfrak{q}}(A) \equiv 0 \bmod \mathbf{Z}^{19)}$ the number $m$ of primes $q \in M$ is even. To every non-empty subset $N \subset M$ which consists of an even number of primes $q$ there exists one and only one quaternion algebra $A(N)$ with the primes in $N$ as its ramification primes. ${ }^{19)}$ In particular, $A=A(M)$. These quaternion algebras generate a subgroup of $\mathfrak{B}(K)$ of order $2^{m-1}$. We claim that this subgroup coincides with $\widetilde{B}(F / K)$. Let $\mathfrak{p}$ be a prime of $F / K$. Then $F \mathfrak{p}$ splits $A$, hence the $\mathfrak{q}$-adic completion $(F \mathfrak{p})_{\mathfrak{q}}$ splits $A_{\mathfrak{q}}=A \underset{K}{\otimes} K_{\mathfrak{q}}$ for every $\mathfrak{q}$. Hence $\left((F p)_{q}: K_{q}\right) \equiv 0 \bmod 2$ for $q \in M$. In particular, this holds for $q \in N$. Hence $(F \mathfrak{p})_{\mathfrak{q}}$ splits $A(N)_{\mathfrak{q}}$. Since this is true for all $\mathfrak{q}$, it follows ${ }^{20)}$ that $F \mathfrak{p}$ splits $A(N)$. Hence $A(N) \in \widetilde{\mathfrak{B}}(F / K)$ for all $N$. Conversely, let $B \in \widetilde{\mathfrak{B}}(F / K)$, $B \neq 1$. Let $L / K$ be a finite algebraic splitting field of $A$. Since $F=F(A)$ is a generic splitting field for $A$, there is a place $\mathfrak{p}$ of $F / K$ such that $F p \subset L .^{21)}$ Since $F p$ splits $B$ it follows that $L$ splits $B$. Hence every finite algebraic splitting field $L$ of $A$ is also a splitting field for $B$. According to the existence theorem of Grunwald ${ }^{22)}$ there exists a finite algebraic extension field $L / K$ such that $\left(L_{\mathfrak{q}}: K_{\mathfrak{q}}\right)=2$ for $q \in M$ and $L_{q_{0}}=K_{q_{0}}$ if $q_{0} \notin M$ is arbitrarily chosen. This field $L$ splits $A$ by construction and hence $B$.

\footnotetext{
18) [9], page 414, theorem 5 .

19) Deuring [6], page 119, Satz 9.

20) Deuring [6], page 117, Satz 1.

21) [9] page 413, theorem 2 .

22) Hasse [7], page 40, Ganz schwacher Existenzsatz.
} 
It follows $\operatorname{inv}_{\mathfrak{q}}(B) \equiv 0 \bmod \frac{1}{2}$ for $q \in M$ and $\operatorname{inv}_{q_{0}}(B)=1$. Since $q_{0} \notin M$ is arbitrary, we see that $B$ is unramified outside of $M$. For $q \in M$, the invariant $\operatorname{inv}_{\mathfrak{q}}(B)$ is either 0 or $\frac{1}{2} \bmod \mathbf{z}$. Hence $B=A(N)$ is a quaternion algebra belonging to some subset $N \subset M$.

We have now shown that $\mathcal{B}(F / K)$ is of order 2 while $\widetilde{B}(F / K)$ is of order $2^{m-1}$. If we choose $A$ such that the number $m$ of ramification points of $A$ is $m>2$, which is possible ${ }^{19)}$, then for the field $F=F(A)$ we have $B(F / K) \neq \widetilde{\mathfrak{B}}(F /$ $K)$.

(b) Examples of function fields $F / K$ over a number field $K$ such that $d(F / K) \neq 1$ and $\mathrm{B}(F / K)=1$.

Let $K$ be a number field and $F / K$ a function field of one variable and genus 1 with the property that $d(F / K)>1$ but $d\left(F_{\mathfrak{q}} / K_{\mathfrak{q}}\right)=1$ for all primes $q$ of $K$, where $F_{\mathrm{q}}=F K_{\mathrm{q}}$ is the constant extension of $F / K$ with respect to the completion $K_{\mathfrak{q}}$ of $\mathfrak{q}^{23)}$ If $A \in \mathfrak{B}(F / K)$, then for every $\mathfrak{q}$ the completion $A_{\mathfrak{q}}$ is split by $F_{\mathfrak{q}} / K_{\mathfrak{q}}$. Since $d\left(F_{\mathfrak{q}} / K_{\mathfrak{q}}\right)=1$ it follows from theorem 1 that $A_{\mathfrak{q}}$ splits too. Hence $\operatorname{inv}_{\mathfrak{q}}(A)$ $\equiv 0 \bmod \mathbf{Z}$ for all q, i.e. $A=1$. $^{19)}$

\section{REFERENCES}

[1] Artin, Kohomologietheorie endlicher Gruppen; Vorlesungsausarbeitung Hamburg, (1957).

[2] Artin-Nesbitt-Thrall, Rings with minimum condition, Ann Arbor (1944).

[ 3 ] Cartan-Eilenberg, Homological Algebra, Princeton, (1956).

[4] Cassels, Arithmetic on Curves of genus one. VI. The Tate-Šafarevič group can be arbitrarily large, Journal für die reine und angewandte Mathematik, vol. 214/215 (1964), 65-70.

[5] Chevalley, Introduction to the theory of functions of one variable, New York, (1951).

[6] Deuring, Algebren, Berlin, (1935).

[7] Hasse, Zum Existenzsatz von Grunwald in der Klassenkörpertheorie, Journal für die reine und angewandte Mathematik, vol. 188 (1950), 40-64.

[8] Reichardt, Einige im Kleinen überall lösbare, im Grossen unlösbare diophantische Gleichungen; Journal für die reine und angewandte Mathematik vol. 184 (1942), $12-18$.

[9] Roquette, On the Galois cohomology of the projective linear group and its applications to the construction of generic splitting fields of algebras; Mathematische Annalen, vol. 150 (1963), 411-439.

23) The existence of such function fields has been proved by Reichardt [8]. See also Cassels [4], page 65, theorem. 
[10] Serre, Corps locaux, Paris, (1962).

[11] Tate, WC-groups over p-adic fields; Séminaire Bourbaki no. 156, Paris, (1957).

[12] Witt, Zerlegung reeller algebraischer Funktionen in Quadrate. Schiefkörper über reellem Funktionenkörper. Journal für die reine und angewandte Mathematik, vol. 171 (1934), 4-11.

[13] Witt, Über ein Gegenbeispiel zum Normensatz, Mathematische Zeitschrift, vol. 39 (1935), 492-467.

[14] Witt, Schiefkörper über diskret bewerteten Körpern. Journal für die reine und angewandte Mathematik, vol. 176 (1937), 153-156.

[15] Zariski-Samuel, Commutative Algebra, vol. II, Princeton, (1960).

Tübingen University

Germany 